Document downloaded from:

http://hdl.handle.net/10251/177479

This paper must be cited as:

Pereira, JMB.; Sartiano, D.; Hervás, J.; Barrera, D.; Madrigal-Madrigal, J.; Sales Maicas, S.; Laurell, F.... (2020). High-voltage Sensor Based on Fiber Bragg Grating in Fibers with Electrodes. IEEE. 1-2. http://hdl.handle.net/10251/177479

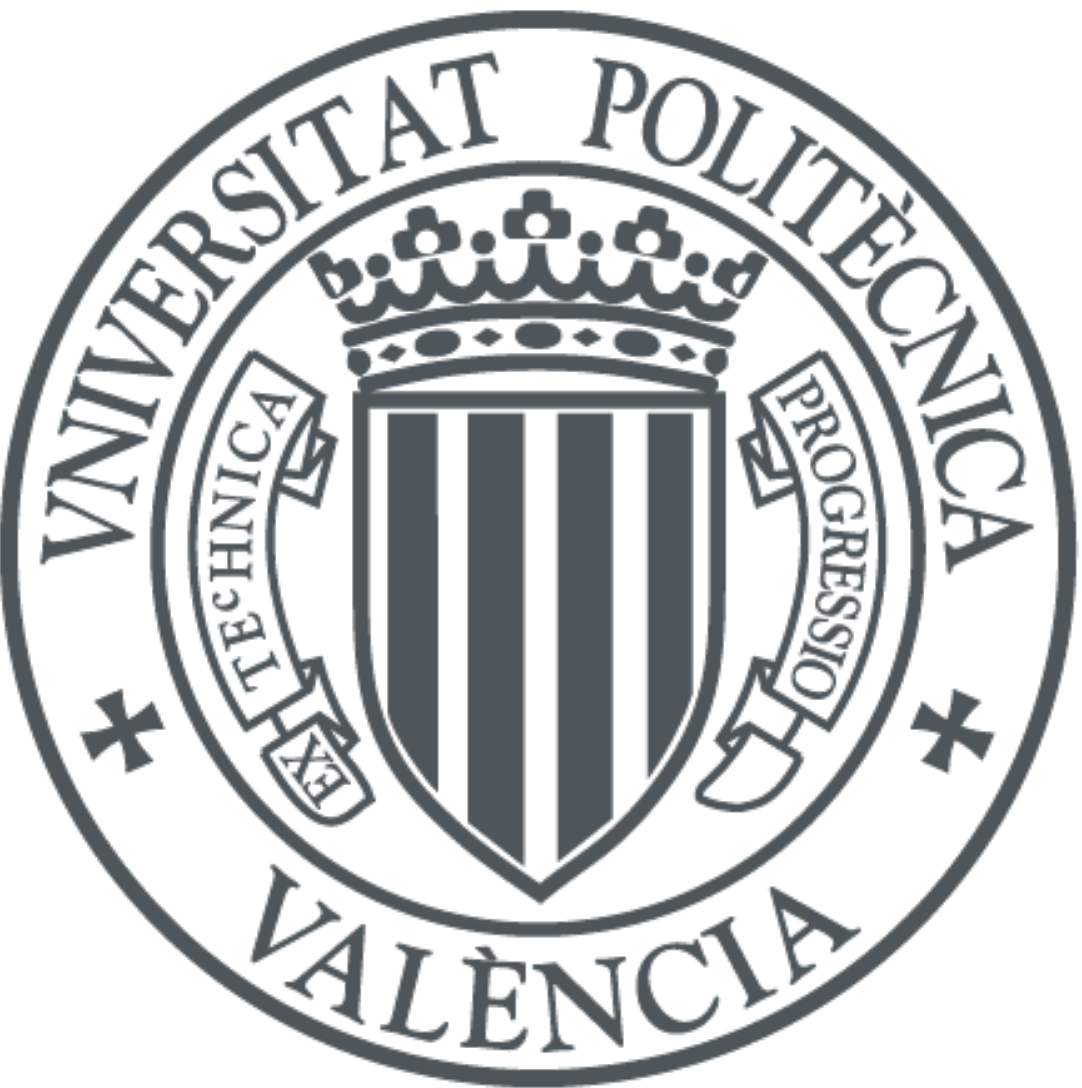

The final publication is available at

https://ieeexplore.ieee.org/abstract/document/9193658

Copyright IEEE

Additional Information 


\title{
High-voltage Sensor Based on Fiber Bragg Grating in Fibers with Electrodes
}

\author{
J. M. B. Pereira ${ }^{1,2}$, D. Sartiano ${ }^{3}$, J. Hervás ${ }^{3}$, D. Barrera ${ }^{3,4}$, J. Madrigal $^{3}$, S. Sales ${ }^{3}$, F. Laurell ${ }^{2}$, O. Tarasenko ${ }^{1}$, \\ W. Margulis ${ }^{1,2}$ \\ ${ }^{1}$ Fiber Optics, RISE Acreo, Electrum 236, 16440, Kista, Sweden. \\ ${ }^{2}$ Dep. of App. Phys., KTH, Roslagstullsbacken 21, 10691, Stockholm, Sweden. \\ 3 iTEAM Research Institute, Universitat Politècnica de València, Valencia 46022, Spain. \\ ${ }^{4}$ Dept. of Electronics, University of Alcalá, Alcalá de Henares, 28805, Spain. \\ Authore-mail address: joao.pereira@ri.se
}

\begin{abstract}
This work describes the use of FBGs inscribed in optical fiber with electrodes for voltage sensing. The results show a quadratic voltage dependence. The device can be explored for a multipoint, single-ended voltage sensing device. (C) 2020 The Authors.

OCIS codes: (060.2340) Fiber Optics components, (060.2370) Fiber Optics sensors, (060.3735) Fiber Bragg gratings.
\end{abstract}

\section{Introduction}

Fiber Bragg Gratings (FBGs) are normally used to monitor changes in temperature and to strain. The sensitivity to strain is often used to indirectly measure other physical parameters such as pressure, vibration, and many others [1]. This work explores the possibility of using FBGs inscribed in fiber with internal electrodes to sense voltage. The Bragg wavelength shift in an FBG is related to refractive index changes in the core, and to the strain [1].

This work shows a proof-of-concept study to use FBGs inscribed in optical fibers to detect voltage. The wavelength shift is related to the voltage applied to the optical fiber electrodes. The proposed experiment opens possibilities for a multiplexed multi-point voltage sensor using optical fibers. For multiplexing, each sensor is a piece of fiber with electrodes, and one FBG inscribed. The sensors can be spliced to SMF28 and separated by several meters.

\section{Methodology}

Applying voltage to the fiber electrodes lead to refractive index changes that are related to the photo-elastic, thermooptic, and electro-optic effect. The electro-optic contribution in a Kerr media is quadratic with the applied electric field [2]. The strain changes can be caused by the electrostatic force between the electrodes, acoustic effects (piezoelectric, and electrostrictive), and thermal effects. The piezoelectric effect is forbidden in symmetric materials like silica [2]. The electrostrictive effect is significant when the modulation frequency is near the resonance frequency of the material which is in the $\mathrm{MHz}$ range for silica [2]. The temperature changes are associated with the power dissipated by the device, and it is dependent on the electric current. In this experiment, the electric current that crosses the device is negligible, and the frequency is relatively low $(20 \mathrm{~Hz})$. With the considerations, the wavelength shift is caused by electro-optic, and photo-elastic response.

The optical fiber used is $125 \mu \mathrm{m}$ twin-hole fiber with $8 \mu \mathrm{m}$ core and symmetrical holes of $28 \mu \mathrm{m}$ separated by 40.8 $\mu \mathrm{m}$. The electrodes were made pumping melted BiSn into the fiber. The fiber was polished from the side and thin tungsten wires were inserted to make the electric contacts. The samples had $0.24 \mathrm{~m}$ long BiSn electrodes and was hydrogen loaded for FBG inscription with phase mask technique. The FBGs used in the experiments are $\sim 1 \mathrm{~cm}$ long.

The interrogation system is in Figure 1-a. The experiment consists of tuning the laser to the quadrature point of the FBG and apply an AC voltage to the fiber electrodes. The AC voltage will result in a periodic wavelength shift that will change the intensity of the signal detected in an oscilloscope.
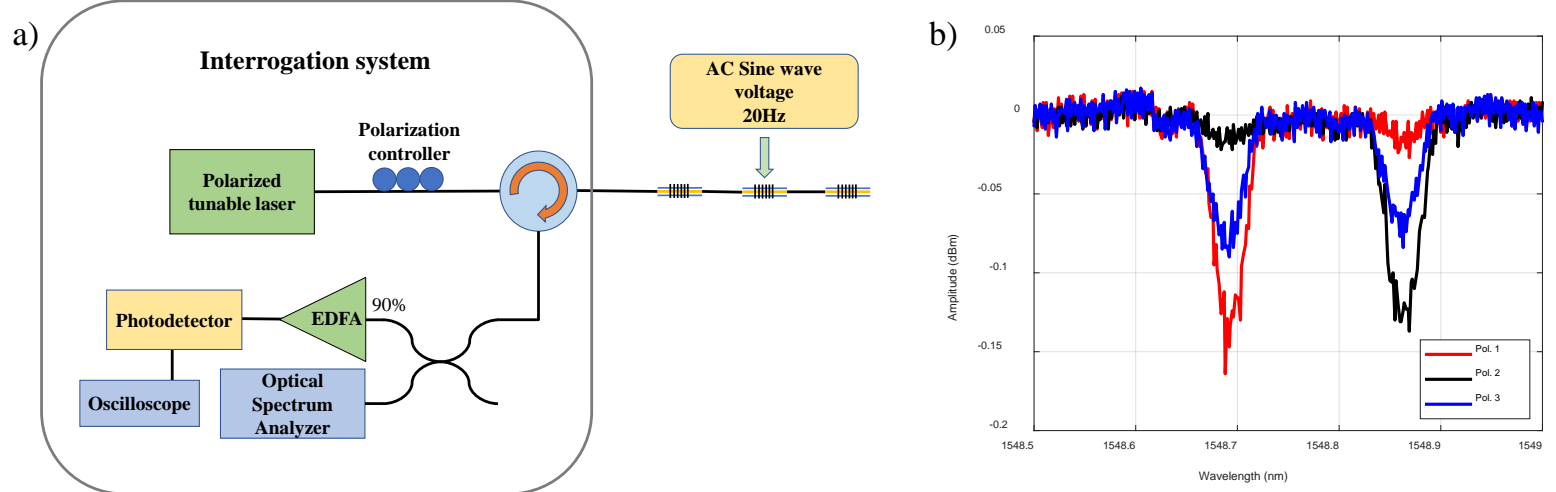

Figure 1 - a) Interrogation system utilized to detect voltage applied to fiber electrodes using an FBG. b) A transmitted spectrum of the FBG utilized in three different polarizations, showing birefringence. 
The transmitted spectrum measured with an OSA for the FBG sample used in the experiment can be seen in Figure 1b. The fact that there are two different FBGs for different polarizations shows that the fiber with electrodes is birefringent.

\section{Results}

The photodetector response when $1500 \mathrm{~V}$ peak to peak and $20 \mathrm{~Hz}$ was applied to fiber electrodes can be seen in Figure 2-a. The FBG shifts to the right for negative, and for positive voltages. When the applied voltage is $0 \mathrm{~V}$ the PD signal is maximum, and when the voltage increase, or decrease the PD signal always decrease. In Figure 2-b a plot with the photodetector voltage versus the applied voltage is shown. A polynomial fit of order two was calculated, and one can see that the parabola is not symmetric around zero volts.

a)

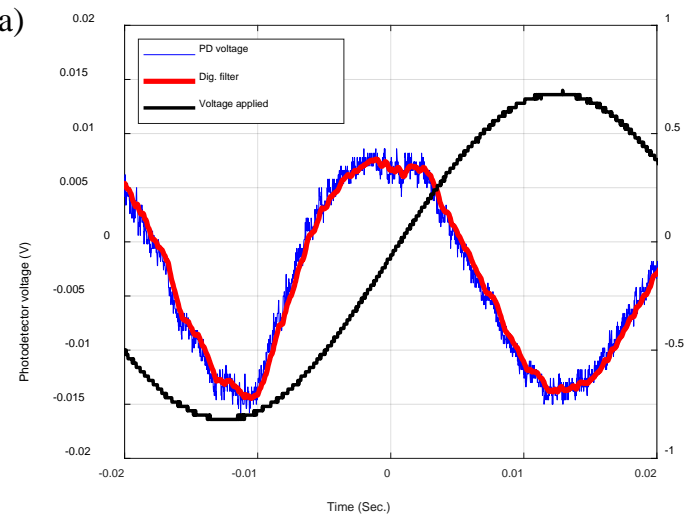

b)

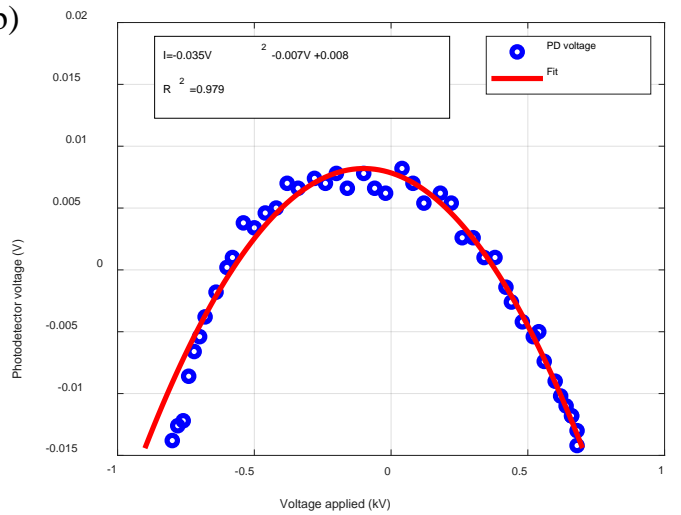

Figure $1-$ a) The blue trace is the photodetector response when voltage is applied to the fiber electrodes. The red curve is a digital filter. In black is the applied peak to peak voltage of $1500 \mathrm{~V}$ and $20 \mathrm{~Hz}$. b) Experimental data, and polynomial fit of the PD voltage versus applied voltage.

The results show that the wavelength shifts depend on the voltage squared. Another observation is that the signal frequency in the photodetector is doubled in relation to the frequency applied. The photodetector output was coupled AC to the oscilloscope. The experiment was performed when the tunable laser was tuned to the left side of the FBG in polarization 1 (Figure 1-b).

\section{Conclusion}

We demonstrated a technique to use FBG inscribed in optical fibers with electrodes for remote voltage sensing. The advantage of the method described is the ability to multiplex voltage sensors that could be separated by many kilometers connected in one open-ended fiber. Each sensor is a piece of fiber with electrodes and one FBG inscribed. This device could be used to monitor voltage without losses in environments where long, and multiple wires cannot be used. Application examples could be power plants or transmission lines.

The results show a quadratic dependence of the voltage applied, but a better understanding of the operation principle needs to be carried to identify, and separate the influences of the electro-optic effect, and photo-elastic effect. The result also shows a phase shift effect that could be caused by a charging effect. This still a work in progress and the experiment can be improved in many ways: The use of a poled fiber could lead to an enhanced, and linear response [3], but with the advantage of not using a closed arrangement in an interferometric setup. Synchronous detection with a lock-in amplifier can improve the SNR.

\section{References}

[1] Kashyap, R., Fiber Bragg Gratings (Second Edition) (Academic Press, 2010).

[2] Alice C. Liu, Michel J. F. Digonnet, and Gordon S. Kino, “Measurement of the dc Kerr and electrostrictive phase modulation in silica," JOSA B, 18 (2), 187-194, (2001).

[3] Michie, A., Bassett I. and Haywood, J., "Electric field and voltage sensing using thermally poled silica fibre with a simple low coherence interferometer,” Meas. Sci. Technol., (17), (2006).

\section{Acknowledgments}

The authors acknowledge financial support from the FINESSE project. FINESSE is funded by the European Union's Horizon 2020 research and innovation programme under the Marie Sklodowska - Curie grant agreement $n^{\circ} 722509$. Partial funding from K. A. Wallenberg Foundation and the Swedish Science Council is gratefully acknowledged. We also thank Kenny Hey Tow (RISE, Sweden) for useful discussions. 